\title{
Retinal Vessel Extraction by Matched Filter with First-Order Derivative of Gaussian
}

\author{
Bob Zhang ${ }^{1}$, Lin Zhang ${ }^{2}$, Lei Zhang ${ }^{2 *}$ and Fakhri Karray ${ }^{1}$ \\ ${ }^{1}$ Dept. of Electrical and Computer Engineering, University of Waterloo \\ Waterloo, ON, Canada, N2L 3G1 \\ ${ }^{2}$ Biometrics Research Center, The Hong Kong Polytechnic University, Hong Kong, China
}

\begin{abstract}
:
Accurate extraction of retinal blood vessels is an important task in computer aided diagnosis of retinopathy. The Matched Filter (MF) is a simple yet effective method for vessel extraction. However, a MF will respond not only to vessels but also to non-vessel edges. This will lead to frequent false vessel detection. In this paper we propose a novel extension of the MF approach, namely the MF-FDOG, to detect retinal blood vessels. The proposed MF-FDOG is composed of the original MF, which is a zero-mean Gaussian function, and the first-order derivative of Gaussian (FDOG). The vessels are detected by thresholding the retinal image's response to the MF, while the threshold is adjusted by the image's response to the FDOG. The proposed MF-FDOG method is very simple; however, it reduces significantly the false detections produced by the original MF and detects many fine vessels that are missed by the MF. It achieves competitive vessel detection results as compared with those state-of-the-art schemes but with much lower complexity. In addition, it performs well at extracting vessels from pathological retinal images.
\end{abstract}

Keywords: retinal image segmentation; vessel detection; matched filter; line detection

\footnotetext{
*Corresponding author. Email: cslzhang@comp.polyu.edu.hk.

This work is supported by the Hong Kong RGC General Research Fund (PolyU 5351/08E).
} 


\section{Introduction}

The automated extraction of blood vessels in retinal images is an important step in computer aided diagnosis and treatment of diabetic retinopathy [1-8], hypertension [10], glaucoma [11], obesity [12], arteriosclerosis and retinal artery occlusion, etc. Vessel extraction is basically a kind of line detection problem, and many methods have been proposed. A class of popular approaches to vessel segmentation are filtering-based methods [5, 9, 18-21] which work by maximizing the response to vessel-like structures. Mathematical morphology [7, 13, 22] is another type of approach by applying morphological operators. Trace-based methods [14] map-out the global network of blood vessels after edge detection by tracing out the center lines of vessels. Such methods rely heavily on the result of edge detection. Machine-Learning based methods [1, 3, 14-15] have also been proposed and they can be divided into two subgroups: supervised methods $[1,3,15]$ and unsupervised methods $[14,16]$. Supervised methods exploit some prior labeling information to decide whether a pixel belongs to a vessel or not, while unsupervised methods do the vessel segmentation without any prior labeling knowledge.

Among the various retinal vessel extraction methods, the classical matched filter (MF) [9] method is a representative one and it has advantages of simplicity and effectiveness. The MF detects vessels by simply filtering and thresholding the original image. Considering the fact that the cross-section of a vessel can be modeled as a Gaussian function, a series of Gaussian-shaped filters can be used to "match" the vessels for detection. However, the MF will have strong responses to not only vessels but also non-vessel edges, for example, the edges of bright blobs and red lesions in retinal images. Therefore, after thresholding the response image, many false detections can result. 
Although the MF employs the prior knowledge that the cross-section of a vessel in a retinal image is Gaussian shaped, it does not fully exploit other information of the vessel profile, in particular that the Gaussian shaped cross section is symmetric with respect to its peak position. If this property can be properly used, it is possible to distinguish the symmetric vessel structures from those asymmetrical non-vessel edges (e.g. the step edge) in a simple but efficient way, and hence the vessel extraction accuracy can be improved.

To this end, in this paper we propose a novel method, namely the matched filter with first-order derivative of the Gaussian (MF-FDOG), as an extension and generalization of the MF. Considering that the cross section of a vessel is a symmetric Gaussian function, we use a pair of filters, the zero-mean Gaussian filter (i.e. the MF) and the first-order derivative of the Gaussian (FDOG), to detect the vessels. For a true vessel, it will have a strong response to the MF around its peak position, while the local mean of its response to the FDOG will be close to zero around the peak position. In contrast, for non-vessel structures, for example the step edge, it will have high response to the MF but the local mean of its response to the FDOG will also be high. Such a difference implies that the vessels and non-vessel edges can be better distinguished by using the MF-FDOG than by using the MF.

By applying the MF-FDOG filters to the retinal image, two response maps, $H$ (by the MF) and $D$ (by the FDOG) can be obtained. The vessel map is detected by applying a threshold $T$ to $H$, while the threshold $T$ is adjusted by $D$ so as to remove the non-vessel edges and extract the fine vessels. As a filtering-based method, the proposed MF-FDOG preserves the simplicity of the original MF; however, it could achieve much higher vessel detection accuracy than the MF, and even comparable to the results of state-of-the-art 
methods [1-2, 7, 13, 18], which have much higher complexity than the MF-FDOG.

The rest of this paper is organized as follows. Section 2 briefly reviews the MF. Section 3 presents the proposed MF-FDOG scheme. Section 4 presents experimental results, and Section 5 concludes the paper.

\section{The Matched Filter}

The Matched Filter (MF) was first proposed in [9] to detect vessels in retinal images. It makes use of the prior knowledge that the cross-section of the vessels can be approximated by a Gaussian function. Therefore, a Gaussian-shaped filter can be used to "match" the vessels for detection. The MF is defined as

$$
f(x, y)=\frac{1}{\sqrt{2 \pi} s} \exp \left(-\frac{x^{2}}{2 s^{2}}\right)-m, \text { for }|x| \leq t \cdot s, \quad|y| \leq L / 2
$$

where $s$ represents the scale of the filter, $m=\left(\int_{-t s}^{t s} \frac{1}{\sqrt{2 \pi s}} \exp \left(\frac{-x^{2}}{2 s^{2}}\right) d x\right) /(2 t s)$ is used to normalize the mean value of the filter to 0 so that the smooth background can be removed after filtering, and $L$ is the length of the neighborhood along the $y$-axis to smooth noise; the criterion $t$ is a constant and is usually set to 3 because more than $99 \%$ of the area under the Gaussian curve lies within the range [-3s, 3s]. The parameter $L$ is also chosen based on s. When s is small, $L$ is set relatively small, and vice versa. In the implementation, $f(x, y)$ will be rotated to detect the vessels of different orientations.

The simplicity of the MF makes it popular in vessel detection. However, a well-known problem of the approach is that it responds not only to vessels but also to non-vessel edges. Figs. 1 (a) (b) illustrate this problem by showing the responses of the MF to a Gaussian function (i.e. the cross-section of a vessel) and an ideal step edge. We can see clearly that 
the MF has strong responses to both the vessel and the step edge. After thresholding, both the vessel and the non-vessel edge will be detected. Therefore, the aim of this paper is to find a simple filtering technique to distinguish the vessels from non-vessel step edges.

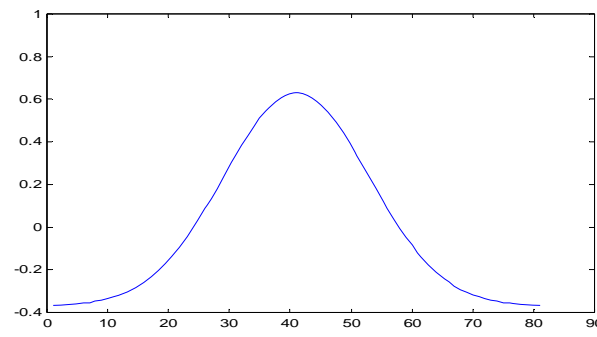

(b-1)

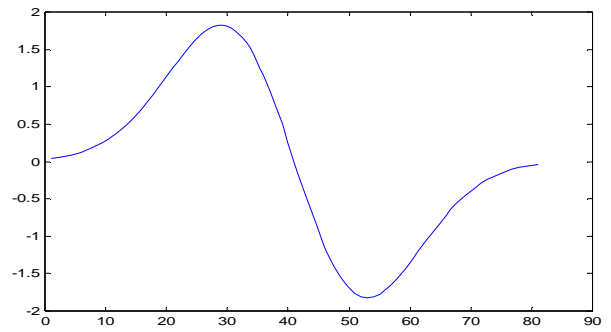

(c-1)

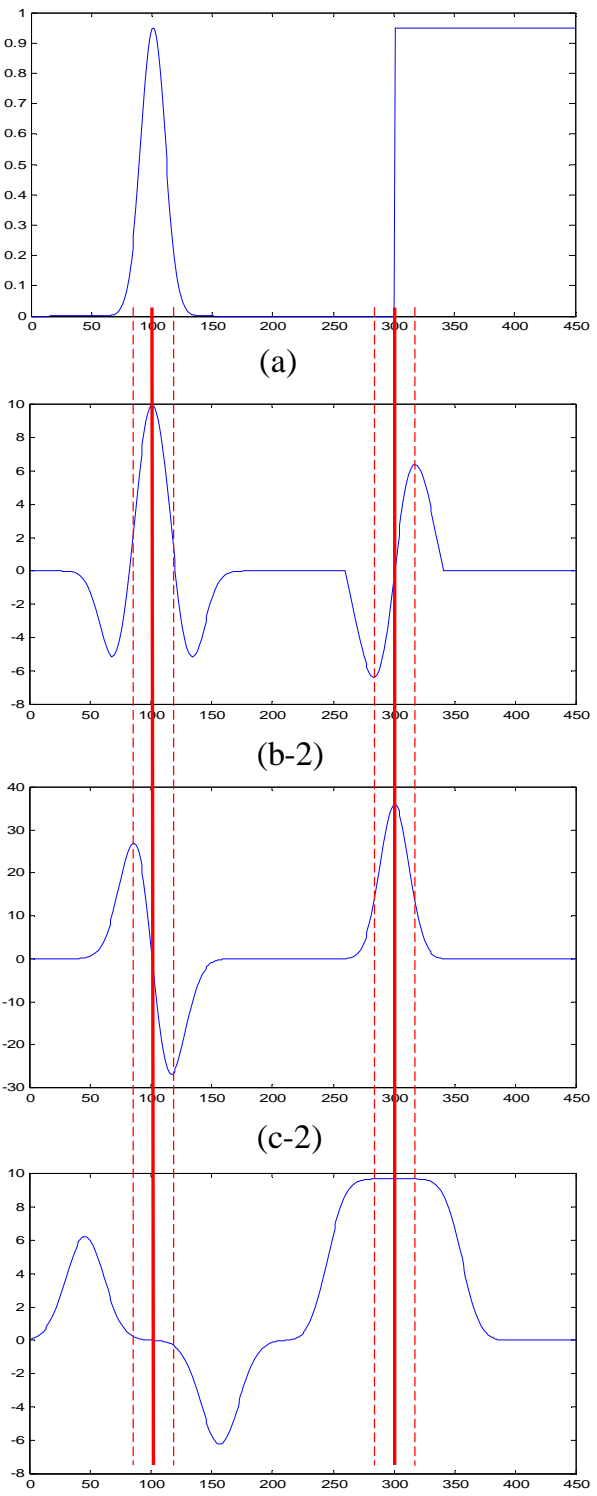

(d)

Figure 1. Responses of the MF and the FDOG to a Gaussian line cross-section and an ideal step edge. (a) A Gaussian line cross-section and an ideal step edge; (b-1) the MF and (b-2) its filter response; (c-1) the FDOG and (c-2) its filter response; (d) the local mean of the response to the FDOG. 


\section{The MF-FDOG}

As we can see in Section 2, the MF has strong responses to both vessels and step edges because it can "match" the shape of both vessels and step edges to some extent. Thus it is hard to distinguish the two types of structures only by the response of MF. Based on the fact that the vessel cross-section is a symmetric Gaussian function while the step edge is asymmetric, we propose a simple scheme by using a pair of filters, instead of only one filter, to distinguish Gaussian vessel structures from non-vessel edges.

The MF is a zero-mean Gaussian filter and it is defined in (1). It can be readily derived that the first-order derivative of the Gaussian (FDOG) is:

$$
g(x, y)=-\frac{x}{\sqrt{2 \pi} s^{3}} \exp \left(-\frac{x^{2}}{2 s^{2}}\right), \quad \text { for }|x| \leq t \cdot s, \quad|y| \leq L / 2
$$

In this paper, we use the MF and the FDOG as "MF-FDOG" for vessel detection. The idea comes from the fact that the Gaussian function (i.e. the cross-section of a vessel) will have a strong positive response to the MF but its response to the FDOG is anti-symmetric. In contrast, although the non-vessel step edge will have partially strong positive responses to MF, its response to the FDOG is positive and symmetric. Fig. 1 shows this by using a synthetic signal. Fig. 1 (a) plots a Gaussian function and an ideal step edge; Figs. 1 (b-1) and (b-2) show the MF and its response to the synthetic signal; Figs. 1 (c-1) and (c-2) show the FDOG and its response. Denote by $h$ the signal's response to the MF. Suppose that we apply a threshold $T$ to $h$ to detect the vessels. Obviously, some of the step edge's responses will be wrongly classified as vessels. However, if we could properly exploit their different responses to the FDOG, as shown in Fig. 1 (c-2), the vessels and non-vessel edges can be better distinguished by thresholding their responses to MF. Unfortunately, in the original FDOG responses, the magnitude around the Gaussian peak (position 100) and the step 
edge (position 300) change rapidly. Therefore, directly using the FDOG response is not robust to tell the two types of structures.

Denote by $d$ the response of the input signal to the FDOG. Let us calculate the local mean of $d$, denoted by $d_{m}$. The local mean value of an element in $d$ is defined as the average of its neighboring elements. Fig. 1 (d) shows the $d_{m}$ of the response $d$ in Fig. 1 (c-2). We can see that in the peak area of the Gaussian function there are strong responses in $h$ (refer to Fig. 1 (b-2)), while the corresponding responses in $d_{m}$ (refer to Fig. 1 (d)) are very low. In contrast, in the neighborhood of the step edge there are also strong responses in $h$ but the corresponding responses in $d_{m}$ are very high. Therefore, the local mean signal $d_{m}$ can be used to adjust the threshold $T$ to detect the true vessels while removing the non-vessel edges. In other words, $T$ should depend on $d_{m}$. If the magnitude in $d_{m}$ is low, this implies that a vessel may appear in the neighborhood, and hence the threshold $T$ applied to $h$ can be small to detect the vessels; if the magnitude in $d_{m}$ is high, this implies that some non-vessel edges may appear, and hence the threshold $T$ can be high to suppress the non-vessel edges.

To this end, we propose a thresholding scheme by using the MF-FDOG for retinal vessel detection. The threshold is applied to the retinal image's response to MF but the threshold level is adjusted by the image's response to FDOG. After filtering the retinal image with the MF-FDOG filters, two response images, $H$ (by the MF) and $D$ (by the FDOG) are obtained ${ }^{1}$. The local mean image of $D$ is calculated by filtering $D$ with a mean filter:

$$
D_{m}=D^{*} W
$$

\footnotetext{
${ }^{1}$ In application, multiple MF-FDOGs along different orientations will be used. For the convenience of discussion, we only consider one MF-FDOG here.
} 
where $W$ is a $w \times w$ filter whose elements are all $1 / w^{2}$. The local mean image $D_{m}$ is then normalized so that each element is within $[0,1]$. We denote by $\bar{D}_{m}$ the normalized image of $D_{m}$.

The threshold $T$ is then set as

$$
T=\left(1+\bar{D}_{m}\right) \cdot T_{c}
$$

where $T_{c}$ is a reference threshold. In this paper, we set $T_{c}$ as follows:

$$
T_{c}=c \cdot \mu_{H}
$$

where $\mu_{H}$ is the mean value of the response image $H$, and $c$ is a constant which can be set between 2 3 based on our experiment experience. By applying $T$ to $H$, the final vessel map $M_{H}$ is obtained as:

$$
\begin{cases}M_{H}=1 & H(x, y) \geq T(x, y) \\ M_{H}=0 & H(x, y)<T(x, y)\end{cases}
$$

It can be seen from (3) (6) that if there is a vessel in the image, then at the corresponding area the magnitude in $\bar{D}_{m}$ will be weak, and hence the threshold $T_{H}$ will be lowered. Thus this vessel can be easily detected by (6). If there are some non-vessel structures in the image, the corresponding magnitude in $\bar{D}_{m}$ will be high, and hence the threshold $T_{H}$ is raised. Thus these non-vessel edges can be suppressed.

We use an example to illustrate the proposed MF-FDOG scheme in Fig. 2. Fig. 2(a) shows an original image "im0001" from the STARE database and it can be clearly seen that there are bright lesions in the middle of the image. Fig. 2(b) shows the response map to MF. It can be seen that MF has strong responses to both vessels and the bright lesions. Fig. 2(c) shows the local mean map of the response to FDOG, i.e. $\bar{D}_{m}$. It can be seen that 
the FDOG response has a higher magnitude (brighter pixels) in the center area where hard exudates are located, while the surrounding vessels produce a lower magnitude (darker pixels). Combining this with the MF response which has higher magnitudes for vessels and lower magnitudes for non-vessels, we can better separate vessel structures from non-vessel structures. Fig. 2(d) is the vessel extraction result of the MF by applying a global threshold to Fig. 2(b), while Fig. 2(e) is the result of the proposed approach. It can be clearly seen that the false detection caused by the bright lesion is greatly reduced, while many fine vessels missed in Fig. 2(d) are detected in Fig. 2(e). The ground truth for this image is shown in Fig. 2(f). We see that the proposed MF-FDOG scheme can more effectively discriminate between true retinal vessels and non-vessel edges than the conventional MF.

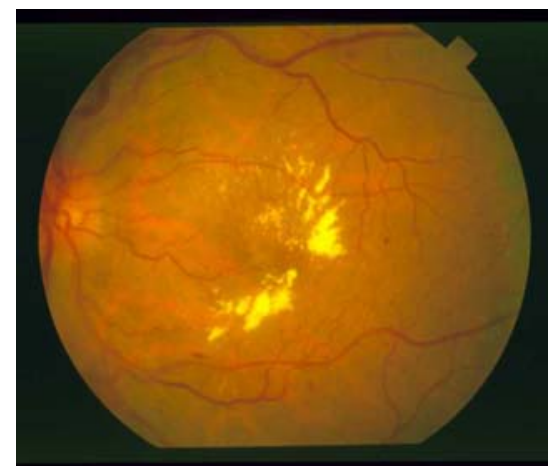

(a)

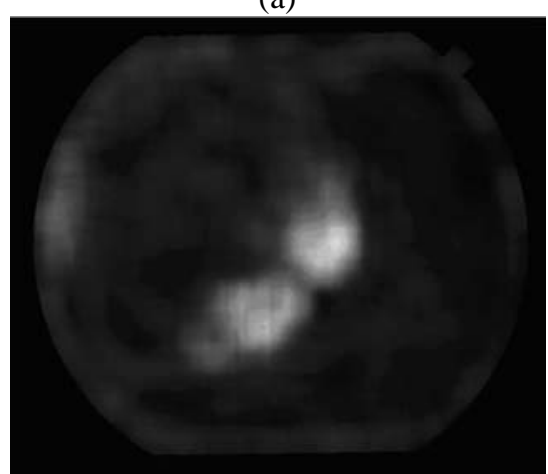

(c)

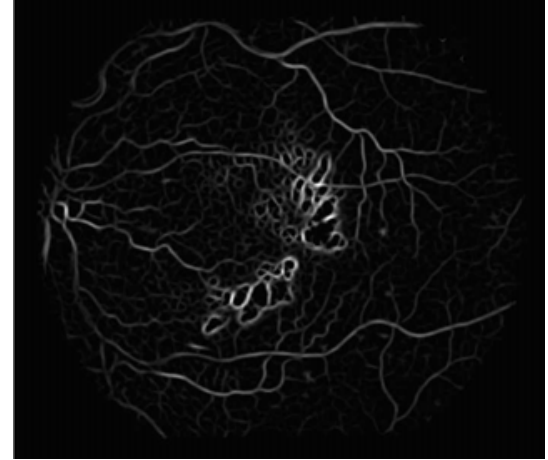

(b)

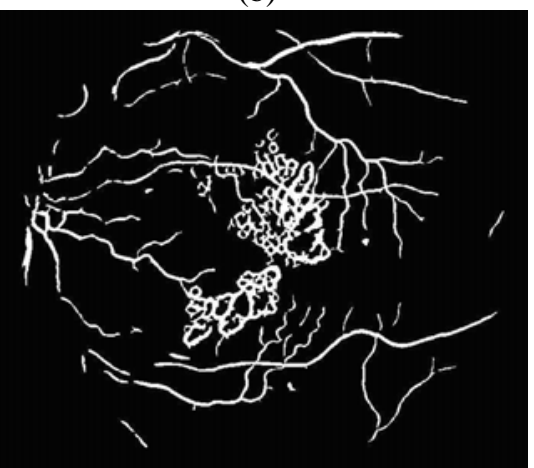

(d) 


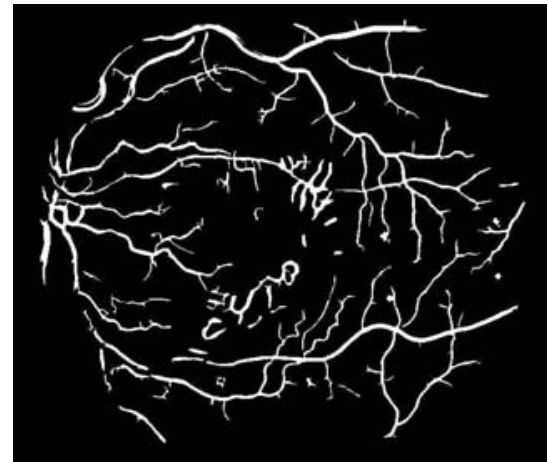

(e)

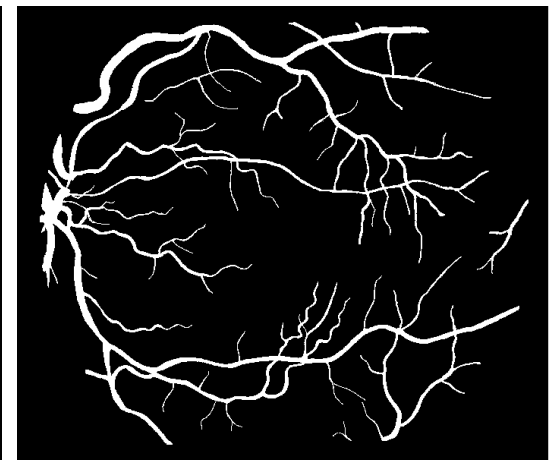

(f)

Figure 2. Illustration of the proposed MF-FDOG retinal vessel extraction scheme. (a) The original image im0001 from the STARE database. (b) The response map to MF. (c) The local mean of the response to FDOG. (d) The vessel extraction result after applying a global threshold to the MF response map. (e) The extraction result of the proposed scheme. (f) The ground truth vessel map.

\section{Experimental Results}

In order to extract both thick and thin vessels in the retinal image, we apply a multi-scale MF-FDOG approach. In other words, we use a large scale to detect thick vessels and a small scale to locate thin vessels. The results of both extractions are then simply combined using the logical OR operation. The key parameters in our experiments are set as follows: $s=1.5$ and $L=9$ (used for wide vessels), $s=1$ and $L=5$ (used for thin vessels) (refer to Eqs. (1) and (2)), $W=31 \times 31$ (refer to Eq. (3)), and $c=2.3$ (refer to Eq. (5)) and 8 directions were used in MF-FDOG filtering. These parameters were chosen based on our experiment experience.

We tested the proposed MF-FDOG method on two publicly available databases, the STARE database [5] and the DRIVE database [1]. The STARE database consists of retinal images captured by the TopCon TRV-50 fundus camera at a $35^{\circ}$ field of view (FOV), which were digitized with 24-bit gray-scale resolution and a spatial resolution of $700 \times 605$ pixels. There are 20 images, ten of which are from healthy ocular fundus and the other ten are from unhealthy ones. The database also provides hand-labeled images as 
the ground truth for vessel segmentation so that the algorithms can be evaluated for comparison. The DRIVE database consists of 40 images captured by the Canon CR5 camera at $45^{\circ} \mathrm{FOV}$, which were digitized at 24 bit with a spatial resolution of $565 \times 584$ pixels. The 40 images were divided into a training set and a test set by the authors of the database. The results of the manual segmentation are available for the two sets. For the images in the test set, a second independent manual segmentation is also available.

To compare different retinal vessel segmentation algorithms, we select (1) detection accuracy, (2) the corresponding TPR (true positive rate), and (3) the FPR (false positive rate) at that accuracy as our performance measures. These performance measures were defined and widely used in literature [1-8, 15]. The detection accuracy is defined as the ratio of the total number of correctly classified pixels to the number of pixels inside the FOV. The TPR is defined as the ratio of the number of correctly classified vessel pixels to the number of total vessel pixels in the ground truth. The FPR is defined as the ratio of the number of non-vessel pixels inside the FOV but classified as vessel pixels, to the number of non-vessel pixels inside FOV in the ground truth.

Table I. Vessel extraction results on the STARE database.

\begin{tabular}{lccc}
\hline Method & TPR & FPR & Accuracy \\
\hline 2nd Human observer & 0.8949 & 0.0610 & 0.9354 \\
Hoover [5] & 0.6751 & 0.0433 & 0.9267 \\
Staal [1] & 0.6970 & 0.0190 & 0.9516 \\
Soares [2] & 0.7165 & 0.0252 & 0.9480 \\
Mendonça [7] & 0.6996 & 0.0270 & 0.9440 \\
Matched filter [9] & 0.6134 & 0.0245 & 0.9384 \\
Martinez-Perez [8] & 0.7506 & 0.0431 & 0.9410 \\
MF-FDOG & $\mathbf{0 . 7 1 7 7}$ & $\mathbf{0 . 0 2 4 7}$ & $\mathbf{0 . 9 4 8 4}$ \\
\hline
\end{tabular}

Table I presents the experimental results on the STARE database by different methods. The performance measures of Staal [1], Mendonça [7] and Martinez-Perez [8] were 
obtained from their original papers. For Soares [2] and Hoover [5], their performance measures were calculated using the segmented images from their websites. The FOV used for the STARE database was generated by the code provided by Soares. All the 20 images in STARE were used in this experiment. (Staal's method [1] used 19 of the 20 images for testing with 10 normal and 9 pathological). The hand-labeled images by the first human expert were used as ground truth. The TPR measure of Martinez-Perez's method [8] is higher than others because it was calculated at a higher FPR level. Overall its accuracy is similar to that of others. To facilitate the comparison of our method with Soares' [2] and Mendonça's [7] methods, we calculated the average TPR corresponding to an FPR of around 0.025 . The experimental results on the STARE database show that the proposed MF-FDOG performs much better than the original MF, also outperforms Hoover's and Mendonça's methods, and is slightly better than Soares' method. It has similar performances to the other state-of-the-art methods but with much less computational cost.

As stated before, one important motivation of the proposed MF-FDOG method is to suppress the false response of the MF to the lesions and blobs that will often appear in the abnormal retinal images. In order to demonstrate the performance of our method in such pathological cases, we compare the results by different methods on the normal and abnormal images in the STARE database in Table $\mathrm{II}^{2}$. Experimental results clearly show that for the abnormal cases, the proposed MF-FDOG method performs significantly better than the MF and Hoover's method, and it achieves better results than Mendonça's and Soares’ methods. Fig. 3 shows an example for visual inspection.

\footnotetext{
${ }^{2}$ Since the result of Staal's method [1] is not available for this experiment, we did not list it in Table II.
} 
Table II. Vessel extraction results on the STARE database (normal versus abnormal cases).

\begin{tabular}{|c|c|c|c|}
\hline Method & TPR & FPR & Accuracy \\
\hline \multicolumn{4}{|c|}{ Normal cases } \\
\hline $2^{\text {nd }}$ Human observer & 0.9646 & 0.0764 & 0.9283 \\
\hline Hoover [5] & 0.6766 & 0.0338 & 0.9324 \\
\hline Mendonça [7] & 0.7258 & 0.0209 & 0.9492 \\
\hline Soares [2] & 0.7554 & 0.0188 & 0.9542 \\
\hline Matched filter[9] & 0.7335 & 0.0218 & 0.9486 \\
\hline MF-FDOG & 0.7526 & 0.0221 & 0.9510 \\
\hline \multicolumn{4}{|c|}{ Abnormal cases } \\
\hline $2^{\text {nd }}$ Human observer & 0.8252 & 0.0456 & 0.9425 \\
\hline Hoover [5] & 0.6736 & 0.0528 & 0.9211 \\
\hline Mendonça[7] & 0.6733 & 0.0331 & 0.9388 \\
\hline Soares[2] & 0.6869 & 0.0318 & 0.9416 \\
\hline Matched filter[9] & 0.5881 & 0.0384 & 0.9276 \\
\hline MF-FDOG & 0.7166 & 0.0327 & 0.9439 \\
\hline
\end{tabular}

Table III. Vessel extraction results for the DRIVE database.

\begin{tabular}{lccc}
\hline Method & TPR & FPR & Accuracy \\
\hline 2nd Human observer & 0.7761 & 0.0275 & 0.9473 \\
Staal [1] & 0.7194 & 0.0227 & 0.9442 \\
Soares [2] & 0.7283 & 0.0212 & 0.9466 \\
Mendonça [7] & 0.7344 & 0.0236 & 0.9452 \\
Matched filter [9] & 0.6168 & 0.0259 & 0.9284 \\
Jiang [6] & - & - & 0.9212 \\
Zana [13] & - & - & 0.9377 \\
Martinez-Perez [8] & 0.7246 & 0.0345 & 0.9344 \\
Garg [16] & - & - & 0.9361 \\
Perfetti [17] & - & - & 0.9261 \\
Cinsdikici [18] & - & - & 0.9293 \\
Al-Rawi [19] & - & - & 0.9510 \\
MF-FDOG & $\mathbf{0 . 7 1 2 0}$ & $\mathbf{0 . 0 2 7 6}$ & $\mathbf{0 . 9 3 8 2}$ \\
\hline
\end{tabular}

Table III presents the results on the DRIVE database. The performance measures of Mendonça [7], Martinez-Perez [8], Garg [16], Perfetti [17], Cinsdikici [18], Al-Rawi [19] were obtained from their original papers while Staal [1] and Soares [2] were calculated using the segmented images from their websites. Jiang's [6] and Zana's [13] methods happened to be published a few years before DRIVE was established, so their results in Table III were implemented by Staal [1] and Niemeijer [3], respectively, with no TPR or FPR given. The DRIVE database provides its own FOV. All 20 images in the test set were 
used in the experiment with the hand-labeled images by the first human expert designated as ground truth. The experimental results on the DRIVE database again validate that the proposed MF-FDOG performs much better than the MF, while it is slightly inferior to some state-of-the-art methods. Fig. 4 illustrates an example of vessel extraction using DRIVE.

To evaluate the proposed MF-FDOG with respect to higher true positives with false positives $<0.05$, we plot the ROC curves for both the DRIVE (dotted line) and STARE (solid line) database in Fig. 5. Each point on the curve represents a different threshold value used to segment the vessels. We can see that the proposed MF-FDOG method has good performance when FP $>0.02$.

The proposed MF-FDOG is competitive with other state-of-the-art methods when using the STARE database (see Table I), and is particularly strong in all three performance measures when dealing with abnormal cases (see Table II). It falls behind some state-of-the-art methods when the DRIVE database is used (see Table III) because the proposed MF-FDOG has advantages in dealing with pathological retinal images but most of the images in the DRIVE test set are normal images from healthy subjects. In addition, some non-vessel structures in retinal images can be very complex and hard to be modeled by step edges. We admit that in such cases our method may fail.

In Table IV we list the running time of our method in comparison with state-of-the-art methods $[2,7]^{3}$. We see that the MF-FDOG requires much less computational cost. Without optimization of the code, it will take about 10 seconds to process one image in the STARE database on a PC with a P-III 1.5 GHz CPU and 512MB RAM.

\footnotetext{
${ }^{3}$ We ran the code of [2] to calculate the running time. The running time of [7] was from the original paper.
} 
Table IV. Running time per image in the STARE database.

\begin{tabular}{llc}
\hline \hline \multicolumn{1}{c}{ Method } & \multicolumn{1}{c}{ System Environment } & Running Time \\
\hline Soares [2] & P-III 1.5GHz, 512 Mb RAM, Matlab & 3.5 minutes \\
Mendonça [7] & P-IV 3.2GHz, 960 Mb RAM, Matlab & 3 minutes \\
MF-FDOG & P-III 1.5GHz, 512 Mb RAM, Matlab & 10 seconds \\
\hline \hline
\end{tabular}

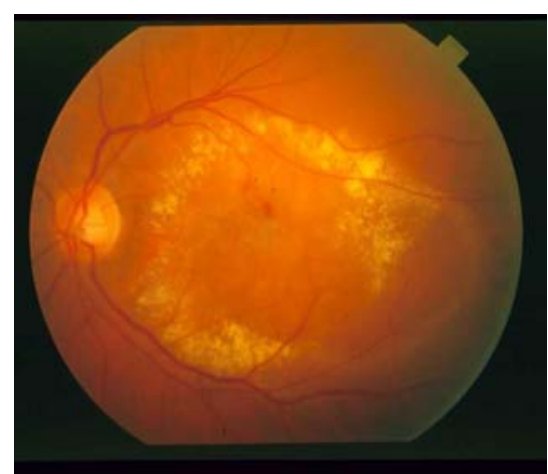

(a)

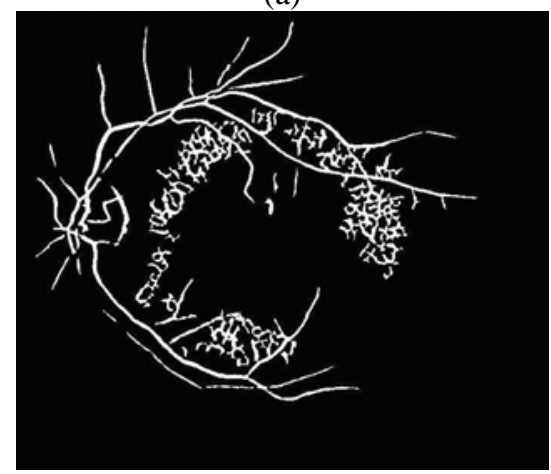

(c)

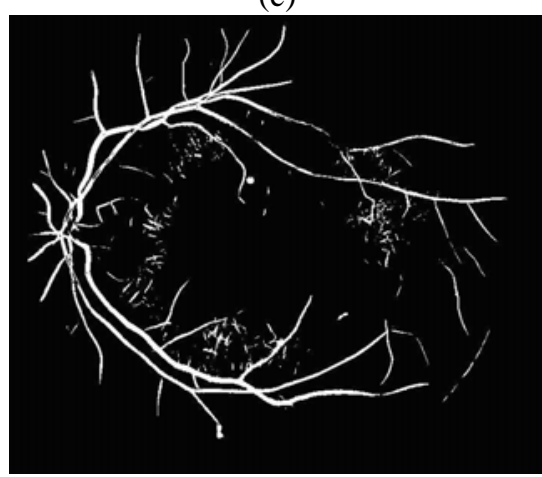

(e)

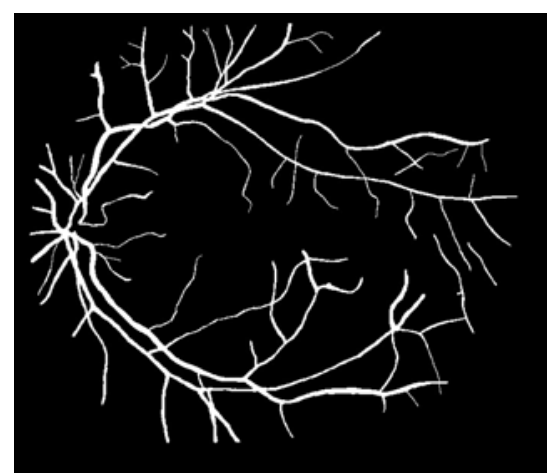

(b)

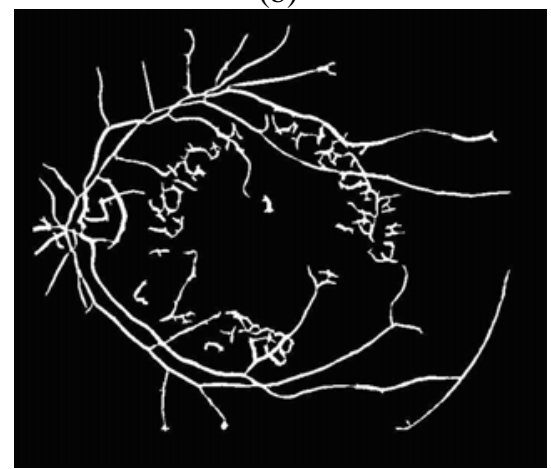

(d)

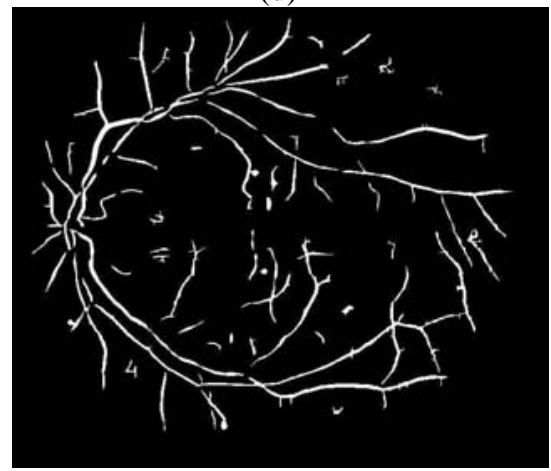

(f)

Figure 3. (a) The original image im0002 from the STARE database; (b) The ground truth vessel map; the vessel extraction results by (c) MF; (d) Hoover [5]; (e) Soares [2]; and (f) the proposed MF-FDOG. 


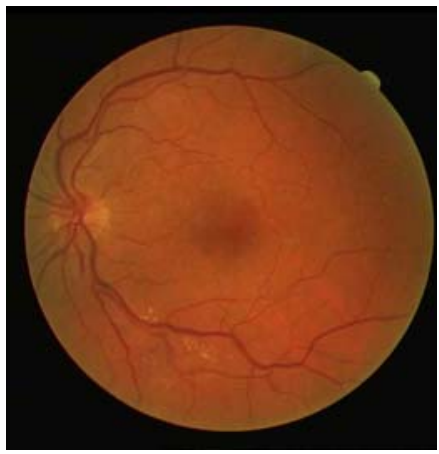

(a)

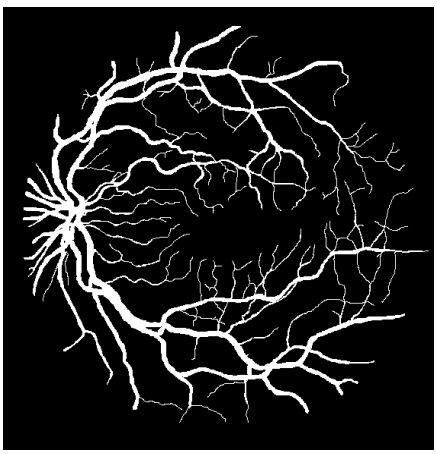

(b)

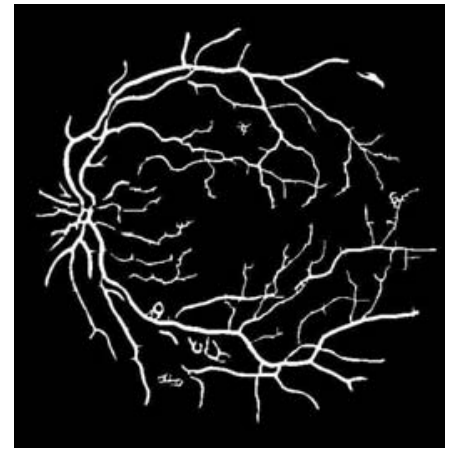

(c)

Figure 4. (a) The original image 3 from the DRIVE database; (b) The ground truth vessel map; the vessel extraction result by (c) the proposed MF-FDOG.

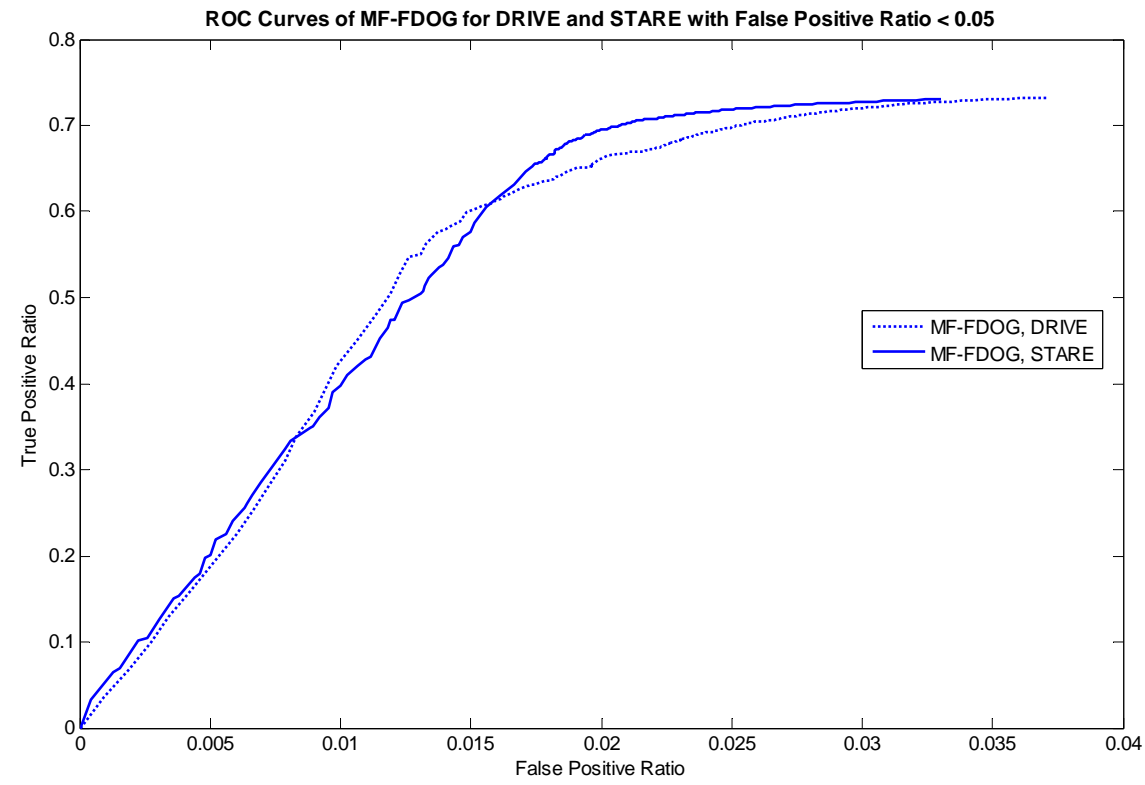

Figure 5. The ROC curves for both STARE and DRIVE when FP $<0.05$ by the proposed method. The solid line represents STARE while the dotted line is DRIVE.

\section{Conclusion}

We proposed a novel retinal blood-vessel extraction method, namely the MF-FDOG, by using both the matched filter (MF) and the first-order-derivative of the Gaussian (FDOG). The retinal vessels were detected by simply thresholding the retinal image's response to the MF but the threshold was adjusted by the image's response to the FDOG. Compared with 
the MF, the MF-FDOG can better distinguish the true vessel structures from non-vessel structures such as blobs and lesions. The experimental results demonstrated that it significantly reduces the false detections generated by the MF and detects many fine vessels that the MF will miss. In particular, the MF-FDOG can extract effectively the vessels in pathological images, leading to competitive results as compared with state-of-the-art schemes; at the same time it has much lower complexity and is much easier to implement.

The use of multiple scales to extract both thick and thin vessels followed by a logical OR to combine the results is effective in general. However, our logical OR operation is not strong enough to remove unwanted structures. Some noisy patterns that exist in either scale can be preserved in the resultant vessel map. This is the weakness of the proposed work. One way to remove the noisy patterns is to employ some post-processing procedures based on their geometric features. Another aspect to be improved for the MF-FDOG is the handling of branching points and the connectivity of vessels. If isolated vessels can be connected to the correct object(s), sensitivity and accuracy will be further improved. We will further investigate these aspects in our future work.

\section{References}

[1] J.J. Staal, M.D. Abramoff, M. Niemeijer, M.A. Viergever, and B. van Ginneken, "Ridge based vessel segmentation in color images of the retina," IEEE Transactions on Medical Imaging, pp. 501-509, 2004.

[2] J.V.B. Soares, J.J.G. Leandro, R.M. Cesar-Jr., H.F. Jelinek, and M.J. Cree, "Retinal vessel segmentation using the 2-d gabor wavelet and supervised classification," IEEE Trans. on Medical Imaging, vol. 25, pp. 1214-1222, 2006.

[3] M. Niemeijer, J.J. Staal, B. van Ginneken, M. Loog, and M.D. Abramoff, “Comparative study of retinal vessel segmentation methods on a new publicly available database”, SPIE Medical Imaging, vol. 5370, pp. 648-656, 2004. 
[4] M. Martínez-Pérez, A. Hughes, A. Stanton, S. Thom, A. Bharath, and K. Parker, "Scale-space analysis for the characterisation of retinal blood vessels," Medical Image Computing and Computer-Assisted Intervention, pp. 90-97, 1999.

[5] A. Hoover, V. Kouznetsova, and M. Goldbaum, "Locating blood vessels in retinal images by piecewise threshold probing of a matched filter response," IEEE Trans. Med. Imag., vol. 19, no. 3, pp. 203-210, 2000.

[6] X. Jiang and D. Mojon, "Adaptive local thresholding by verification based multithreshold probing with application to vessel detection in retinal images," IEEE Trans. Pattern Anal. Mach. Intell., vol. 25, no. 1, pp. 131-137, 2003.

[7] A.M. Mendonca and A. Campilho, "Segmentation of retinal blood vessels by combining the detection of centerlines and morphological reconstruction", IEEE Transactions on Medical Imaging, vol. 25, no. 9, pp.1200-1213, 2006.

[8] M.E. Martinez-Perez, A.D. Hughes, S.A. Thom, A. A. Bharath, and K.H. Parker, "Segmentation of blood vessels from red-free and fluorescein retinal images", Medical Image Analysis, vol. 11, no. 1, pp. 47-61, 2007.

[9] S. Chaudhuri, S. Chatterjee, N. Katz, M. Nelson, and M. Goldbaum, "Detection of blood vessels in retinal images using two-dimensional matched filters," IEEE Trans. Med. Imaging, pp. 263-269, 1989.

[10] H. Leung, J.J. Wang, E. Rochtchina, T.Y. Wong, R. Klein, and P. Mitchell, "Impact of current and past blood pressure on retinal arteriolar diameter in older population," J. Hypertens., pp. 1543-1549, 2003.

[11] P. Mitchell, H. Leung, J.J. Wang, E. Rochtchina, A.J. Lee, T.Y. Wong, and R. Klein, "Retinal vessel diameter and open-angle glaucoma: the Blue Mountains eye study," Ophthalmology, pp. 245-250, 2005.

[12] J.J. Wang, B. Taylor, T.Y. Wong, B. Chua, E. Rochtchina, R. Klein, and P. Mitchell, "Retinal vessel diameters and obesity: a population-based study in older persons," Obes.Res., pp.206-214, 2006.

[13] F. Zana, and J.C. Klein, "Segmentation of vessel-like patterns using mathematical morphology and curvature evaluation,” IEEE Trans. Image Process., pp. 1010-1019, 2001.

[14] Y. Tolias, and S. Panas, "A fuzzy vessel tracking algorithm for retinal images based on fuzzy clustering,” IEEE Trans. Med. Imaging, pp. 263-273, 1998.

[15] C. Sinthanayothin, J. Boyce, and C.T. Williamson, "Automated Localisation of the Optic Disk, Fovea, and Retinal Blood Vessels from Digital Colour Fundus Images,” British Journal of Ophthalmology, pp. 902-910, 1999.

[16] S. Garg, J. Sivaswamy, and S. Chandra, "Unsupervised curvature-based retinal vessel segmentation," Proc. of IEEE International Symposium on Bio-Medical Imaging, pp. 344 347, 2007.

[17] R. Perfetti, E. Ricci, D. Casali, and G. Costantini, "Cellular neural networks with virtual template expansion for retinal vessel segmentation," IEEE Transactions on Circuits and Systems II, vol. 54, pp. 141 - 145, 2007.

[18] M. Cinsdikici, and D. Aydin, "Detection of blood vessels in ophthalmoscope images using MF/ant (matched filter/ant colony) algorithm," Computer Methods and Programs in Biomedicine, vol. 96, pp. 85 - 95, 2009.

[19] M. Al-Rawi, M. Qutaishat, and M. Arrar, "An improvement matched filter for blood vessel detecetion of digital retinal images," Computers in Biology and Medicine, vol. 37, pp. 262 267, 2007.

[20] O. Chutatape, L. Zheng, and S. Krishnan, "Retinal blood vessel detection and tracking by matched Gaussian and kalman filters," Proc. of Engineering in Medicine and Biology Society, pp. 3144 - 3149, 1998. 
[21] R.M. Rangayyan, F.J. Ayres, F. Oloumi, F. Oloumi, and P. Eshghzadeh-Zanjani, "Detection of blood vessels in the retina with multiscale Gabor filters," J. Electron. Imaging, vol. 17, 023018, 2008.

[22] M.M. Fraz, M.Y. Javed, and A. Basit, "Evaluation of Retinal Vessel Segmentation Methodologies Based On Combination of Vessel Centerlines and Morphological Processing," IEEE International Conference on Emerging Technologies, October 18 - 19, Rawalpindi, Pakistan, 2008. 